\title{
Reinforcement and Metacognition
}

\author{
J. Jozefowiez \\ Universidade do Minho \\ J. E. R. Staddon \\ Duke University \\ D. T. Cerutti \\ California State University-East Bay
}

\begin{abstract}
We return to the issue of reinforcement addressed by Smith, Beran, Couchman, Coutinho \& Boomer. We argue that their concerns are unfounded because (a) reinforcing the 'uncertain' response need not undermine an otherwise convincing demonstration of animal metacognition, even for skeptics, (b) with or without metacognition, in the absence of the appropriate reinforcement contingencies, animals will not choose at all.
\end{abstract}

Keywords: Metacognition, comparative metacognition, uncertainty monitoring, metamemory, quantitative modeling.

We return to a central theme of Smith, Beran, Couchman, Coutinho, \& Boomer's contribution, which echoes their earlier concern (Smith, Beran, Couchman, \& Coutinho, 2008), that explicit reinforcement of the uncertain response jeopardizes the quest for animal metacognition by opening the door to a low-level associative approach. These authors argue that procedures using a "pure," because unreinforced, uncertain response should be used instead. Examples of steps in this direction would be Smith, Beran, Redford \& Washburn (2006)'s study where reinforcers are held until the end of the task or Beran, Smith, Redford \& Washburn (2006)'s study where no reinforcement for the uncertain response is provided at all.

We already briefly addressed this issue in our own contribution; we would like to expand on it here. In a nutshell, we think that Smith et al.'s concerns are without foundation.

J. Jozefowiez, Instituto de Educação e Psicologia, Universidade do Minho, Braga, Portugal; J. E. R. Staddon, Department of Psychology and Neuroscience, Duke University, Durham, North Carolina 27708; D.T. Cerutti, Department of Psychology, California State University - East Bay, Hayward, California. Correspondence concerning this article should be addressed to Jeremie Jozefowiez, Instituto de Educação e Psicologia, Universidade do Minho, 4710 Braga, Portugal. E-mail: jeremie@iep.uminho.pt.
First, even for skeptics of animal metacognition like us, reinforcing the uncertain response will not jeopardize what might be a convincing demonstration of animal metacognition. Second, reinforcement of some sort must be involved in any kind of discrimination, metacognitive or otherwise.

To understand our argument, imagine you are participating to a quiz show. Except for behaviorist killjoys like us, most people would agree (perhaps wrongly!) that humans have metacognition - we grant this point here, for the sake of argument. The host of the show tells you: "Which team won the European league championship in 2008: Manchester United or Chelsea? If you give the correct answer, you will win $\$ 1000$. Otherwise, I will just ask you another question." Alternatively, he points out, you can choose the "don't know" (uncertain) option, in which case you just get another question. If, just like the authors of this paper, you have absolutely no interest in European soccer, you will be at that time in a state of maximum uncertainty and, being blessed with metacognition, you will know that you do not know. Yet, obviously, it would not be wise to pick the uncertain response: if you choose to answer the question, you have one chance out of two to win $\$ 1000$ dollars; but by picking the uncertain response, you win nothing, for sure.

Hence, even for an organism endowed with metacogni- 
tive abilities, there must be some incentive for picking the uncertain response. In other words, the uncertain response must be reinforced in one way or another. In the absence of risk aversion, the payoff for the uncertain response must be more than half what the subject would earn by responding randomly to the test question.

Delaying reinforcement by giving trials in blocks with the reinforcement at the end, as Smith et al. (2006) did in an ingenious experiment, doesn't really solve the problem. The procedure simply impairs the correlation between real uncertainty (assessed by actual percent correct) and the animal's assessment of uncertainty (assessed by its choice of the uncertain response). Thus, in Smith et al.'s experiment, the monkeys were equally likely to respond "uncertain" to stimuli that were in fact responded to correctly $95 \%$ and $24 \%$ correct. These data may still be explicable as a sort of winner-take-all competition between a low but nonzero tendency to make the uncertain response (what Crystal \& Foote, in press, term a stimulus-independent hypothesis) competing with the weak tendencies to make either discrimination response in the confusable range.

In other words, any model of the task used to demonstrate animal metacognition must involve the subject making a decision about which response to make based on some information to which it has access. The critical point regarding metacognition is not the process by which the decision is made - which necessarily will involve the reinforcing effects of the payoffs for the various responses available - but the information it uses to make this decision.

To take a parallel example, in studies aimed at demonstrating numerical abilities, the animals obviously perform the way they do because of the contingencies of reinforcement implemented by the experimenter. But this does not affect the real issue, which is: what information does the animal use to perform the task, numerosity or some other stimulus dimension correlated with numerosity but not numerosity itself?

Put in yet another way, because tasks used to demonstrate animal metacognition are discrimination tasks, any account of performance in these tasks will be an associative one where the animal associates reinforcement of a response to specific stimulus values. This is not the issue. The issue is what the stimulus values are. Hence, the fact that monkeys meet the behavioral criteria for metacognition while pigeons do not has no implication about whether or not the monkeys' performance require us to invoke metacognition or can be explained by low-level models. It just shows that both animals are using different kinds of information to make their decisions, even though reinforcement plays a role in both cases. In monkeys, that information is correlated in some way with its chance of success on that trial, i.e., with an effect of its past history with similar (in a way to be discovered) trials. (Of course, sensitivity to past history is also a property of models such as BEM, which do not resort to the faculty of metacognition.) Pigeons, however, apparently do not show this kind of sensitivity (Inman \& Shettleworth, 1999), though which information they use instead is not yet clear.

Moreover, as our game-show example shows, you cannot escape reinforcement: there must be a reason why the subject picks the uncertain response and there is no other candidate but reinforcement. Even though Smith et al. claim to have "provided strong evidence that the reinforcement structure of this task did not determine the animal's uncertainty-response strategy," the question remains: if not reinforcement, then what? How does the monkey set its decision criteria if it does not use reinforcement? It's almost a logical necessity: cherchez le renforçateur, as Crystal and Foote did, and you will find it.

Hence, using complex procedures, such as the one developed by Smith et al. (2006) or Beran et al. (2006), will just make the life of the researchers unnecessarily complicated. First, it may not be possible to use such procedures with other species. It is pretty amazing that one of monkeys from the Smith et al. (2006) study continued to respond in the new task despite the withholding of explicit reinforcers; we doubt that rats or pigeons would be so obliging. Second, complex as it is, the procedure does not eliminate reinforcement. In Crystal and Foote's words, it just makes the reinforcement struc-ture more opaque, making theoretical analysis, whether favorable or not to the idea of animal metacognition, more difficult. Finally, reinforcement is a false problem. The issue is not why the animals decide to pick this response instead of that one, which is where reinforcement is relevant, but what information it uses to make this decision.

\section{References}

Beran, M. J., Smith, J. D., Redford, J. S., \& Washburn, D. A. (2006). Rhesus macaques (Macaca mulatta) monitor uncertainty during numerosity judgments. Journal of Experimental Psychology; Animal Behavior Processes, 32,111-119. doi:10.1037/0097-7403.32.2.111

Crystal, J.D. \& Foote, A.L. (2009). Metacognition in animals. Comparative Cognition and Behavior Reviews, 4, 1-16.

Inman, A., \& Shettleworth, S. J. (1999). Detecting metamemory in nonverbal subjects: a test with pigeons. Journal of Experimental Psychology: Animal Behavior Processes, 25, 389-395. doi:10.1037/0097-7403.25.3.389

Smith, J. D., Beran, M. J., Redford, J. S., \& Washburn, D. A. (2006). Dissociating uncertainty responses and reinforcement signals in the comparative study of uncer- 
tainty monitoring. Journal of Experimental Psychology: General, 135, 282-197

doi:10.1037/0096-3445.135.2.282

Smith, J. D., Beran, M. J., Couchman, J. J., \& Coutinho, M. V. C. (2008). The comparative study of metacognition: sharper paradigms, safer inferences. Psychonomic Bulletin and Review, 15, 679-691. doi:10.3758/PBR.15.4.679

Smith, J.D., Beran, M.J., Couchman, J. J., Coutinho, M. V. C., \& Boomer, J. B. (2009). Animal metacognition: problems and prospect. Comparative Cognition and Behavior Reviews, 4, 50-53.

Staddon, J. E. R., \& Innis, N. K. (1966). Preference for fixed vs. variable amounts of reward. Psychonomic Science, 4, 193-194.

Staddon, J. E. R., Jozefowiez, J., \& Cerutti, D. T. (2007). Metacognition: a problem, not a process. Psycrit, April. (www.psycrit.com). 